\title{
ON THE CHARACTERISTIC WORD OF THE INHOMOGENEOUS BEATTY SEQUENCE
}

\author{
TAKaO KomatsU
}

\begin{abstract}
We detail the sequence $\left(f_{n}\right)$ where $f_{n}=[(n+1) \theta+\phi]-[n \theta+\phi]-[\theta]$. This description of the inhomogeneous Beatty sequence generalises earlier work dealing with special cases in which $\phi$ is restricted to rational values.
\end{abstract}

\section{INTRODUCTION}

The Beatty sequence $([n \theta+\phi])$ of integer parts of $n \theta+\phi, n=1,2, \ldots$ has been studied extensively. In that context it is natural to consider the sequence of differences $f_{1}, f_{2}, f_{3}, \ldots$, where

$$
f_{n}=[(n+1) \theta+\phi]-[n \theta+\phi]-[\theta] .
$$

Plainly each $f_{n}$ is equal to either 0 or 1 . The word $f_{1} f_{2} f_{3} \cdots$ is called the characteristic word, or just word of the sequence.

Stolarsky [9] constructs this characteristic word in the case when $\phi=0$ by using shift operators; and this is generalised by Fraenkel, Muskin and Tassa [3]. On the other hand, van Ravenstein, Winley and Tognetti [8] obtain the word in a special case from the three gap theorem. Danilov [1] had a similar result by a different method. Recently, Nishioka, Shiokawa and Tamura [7] get the word for an irrational $\theta$ and a rational $\phi$ from Mahler functions. However, their result does not match the facts when $\phi \neq 0$ (The correct version is described in [5]). Two other papers dealing with the inhomogeneous case are $[4]$ and $[6]$.

Venkov [10,65-68] rewrites Markov's method to obtain the characteristic word. We apply Venkov's method with the goal of obtaining the word $f_{1} f_{2} f_{3} \ldots$ in the inhomogeneous case.

Received 30th May, 1994

Copyright Clearance Centre, Inc. Serial-fee code: 0004-9729/95 \$A2.00+0.00. 


\section{THE WORD OF $f(n ; \theta, \phi)$}

We write

$$
f_{n}=f(n ; \theta, \phi)=[(n+1) \theta+\phi]-[n \theta+\phi]-[\theta] .
$$

As always, $[\psi]$ denotes the integer part (floor) of $\psi$, and $\{\psi\}=\psi-\lfloor\psi\rfloor$ is its fractional part. We shall assume that $0<\theta<1$.

Venkov details the characteristic of $f(n ; \theta, 0)$ and $f(n ; \theta, 1 / 2)$. We obtain a more general result, that is, with $\theta$ and $\phi$ arbitrary real numbers.

We may take $0<\theta<1$ without loss of generality, because the result is trivial if $\theta$ is an integer. We also let $0 \leqslant \phi<1$. We begin by supposing that $\theta+\phi<1$.

We start from the continued fraction expansion $\theta=\left[0, a_{1}, a_{2}, \ldots\right]=\left[0, a_{1}+\theta_{1}\right]$, where $\theta_{n-1}=\left[0, a_{n}, a_{n+1}, \ldots\right]$. And we also introduce the expansion of $\phi$ in terms of the sequence $\left\{\theta_{0}, \theta_{1}, \ldots\right\}$, that is, by using the notation $\lceil\psi\rceil$, the ceiling of $\psi$

$$
\begin{aligned}
\phi & =b_{0}-\phi_{0}, & b_{0} & =\lceil\phi\rceil \\
\frac{\phi_{n-1}}{\theta_{n-1}} & =b_{n}-\phi_{n}, & b_{n} & =\left\lceil\frac{\phi_{n-1}}{\theta_{n-1}}\right\rceil \quad(n \geqslant 1) .
\end{aligned}
$$

If for $m=1,2, \ldots$ we put $\alpha_{m}=m a_{1}+\left[m \theta_{1}\right]$, then $\left[\alpha_{m} \theta+\phi\right]=m+\left[\phi-\left\{m \theta_{1}\right\} \theta\right]$ and $\left[\left(\alpha_{m}+1\right) \theta+\phi\right]=m+\left[\phi+\left(1-\left\{m \theta_{1}\right\}\right) \theta\right]$. So for any non-negative integer $\lambda_{m}$ we have $\left[\left(\alpha_{m}-\lambda_{m}\right) \theta+\phi\right]=m+\left[\phi-\left(\lambda_{m}+\left\{m \theta_{1}\right\}\right) \theta\right]$.

Noting that $0 \leqslant\left\{m \theta_{1}\right\} \theta<1$ and that $\left(1-\left\{m \theta_{1}\right\}\right) \theta<1-\phi$, it follows that

$$
\begin{aligned}
{\left[\alpha_{m} \theta+\phi\right] } & = \begin{cases}m-1, & \text { if }\left\{m \theta_{1}\right\} \theta>\phi, \\
m, & \text { otherwise },\end{cases} \\
{\left[\left(\alpha_{m}+1\right) \theta+\phi\right] } & =m, \\
{\left[\left(\alpha_{m}-\lambda_{m}\right) \theta+\phi\right] } & = \begin{cases}m-1, & \text { if }\left(\lambda_{m}+\left\{m \theta_{1}\right\}\right) \theta>\phi, \\
m, & \text { otherwise }\end{cases}
\end{aligned}
$$

If $\left\{(m+1) \theta_{1}\right\} \theta>\phi$, then

$$
\begin{gathered}
f_{\alpha_{m}+1}+f_{\alpha_{m}+2}+\cdots+f_{\alpha_{m+1}-1}=\left[\alpha_{m+1} \theta+\phi\right]-\left[\left(\alpha_{m}+1\right) \theta+\phi\right]=m-m=0 \\
f_{\alpha_{m+1}}=\left[\left(\alpha_{m+1}+1\right) \theta+\phi\right]-\left[\alpha_{m+1} \theta+\phi\right]=m+1-m=1 .
\end{gathered}
$$

Thus, $f_{\alpha_{m}+1} f_{\alpha_{m}+2} \cdots f_{\alpha_{m+1}}=\underbrace{00 \cdots \cdots \cdots 0}_{\alpha_{m+1}-\alpha_{m}-1} 1$.

And if $\left\{(m+1) \theta_{1}\right\} \theta \leqslant \phi$,

$$
\begin{gathered}
{\left[\alpha_{m+1} \theta+\phi\right]-\left[\left(\alpha_{m}+1\right) \theta+\phi\right]=m+1-m=1,} \\
{\left[\left(\alpha_{m+1}+1\right) \theta+\phi\right]-\left[\alpha_{m+1} \theta+\phi\right]=m+1-(m+1)=0 .}
\end{gathered}
$$


Thus in this case

$$
\begin{aligned}
& f_{\alpha_{m}+1}+f_{\alpha_{m}+2}+\cdots+f_{\alpha_{m+1}-\lambda_{m+1}-1}=\left[\left(\alpha_{m+1}-\lambda_{m+1}\right) \theta+\phi\right]-\left[\left(\alpha_{m}+1\right) \theta+\phi\right] \\
& = \begin{cases}m-m=0, & \text { if }\left(\lambda_{m+1}+\left\{(m+1) \theta_{1}\right\}\right) \theta>\phi, \\
m+1-m=1, & \text { otherwise } .\end{cases}
\end{aligned}
$$

So, if $\lambda_{m+1}$ is the least non-negative integer satisfying $\left(\lambda_{m+1}+\left\{(m+1) \theta_{1}\right\}\right) \theta>\phi$,

$$
f_{\alpha_{m}+1} f_{\alpha_{m}+2} \cdots f_{\alpha_{m+1}}=\underbrace{00 \cdots \cdots \cdots \cdots}_{\alpha_{m+1}-\alpha_{m}-\lambda_{m+1}-1} 1 \underbrace{0 \cdots 0}_{\lambda_{m+1}} \text {. }
$$

Next, consider $f_{1} f_{2} \cdots f_{a_{1}}$, noting that $\alpha_{1}=a_{1}$. Much as above, we have the following:

$$
\begin{gathered}
{\left[a_{1} \theta+\phi\right]=1+\left[\phi-\theta \theta_{1}\right]= \begin{cases}0, & \text { if } \theta \theta_{1}>\phi \\
1, & \text { otherwise }\end{cases} } \\
{\left[\left(a_{1}+1\right) \theta+\phi\right]=1+\left[\phi+\left(1-\theta_{1}\right) \theta\right]=1}
\end{gathered}
$$

For a non-negative integer $\lambda_{1}$,

$$
\left[\left(a_{1}-\lambda_{1}\right) \theta+\phi\right]=1+\left[\phi-\left(\lambda_{1}-\theta_{1}\right) \theta\right]= \begin{cases}0, & \text { if }\left(\lambda_{1}+\theta_{1}\right) \theta>\phi \\ 1, & \text { otherwise }\end{cases}
$$

If $\theta \theta_{1}>\phi$,

$$
\begin{aligned}
f_{1}+f_{2}+\cdots+f_{a_{1}-1} & =\left[a_{1} \theta+\phi\right]-[\theta+\phi]=0 \\
f_{1}+f_{2}+\cdots+f_{a_{1}} & =\left[\left(a_{1}+1\right) \theta+\phi\right]-[\theta+\phi]=1
\end{aligned}
$$

Thus,

$$
f_{1} f_{2} \cdots f_{a_{1}}=\underbrace{0 \cdots 0}_{a_{1}-1} 1 \text {. }
$$

If $\theta \theta_{1} \leqslant \phi, f_{1}+f_{2}+\cdots+f_{a_{1}-1}=1$ and $f_{1}+f_{2}+\cdots+f_{a_{1}}=0$. So in this case

$$
\begin{aligned}
f_{1}+f_{2}+\cdots+f_{a_{1}-\lambda_{1}-1} & =\left[\left(a_{1}-\lambda_{1}\right) \theta+\phi\right]-[\theta+\phi] \\
& = \begin{cases}0, & \text { if }\left(\lambda_{1}+\theta_{1}\right) \theta>\phi, \\
1, & \text { otherwise. }\end{cases}
\end{aligned}
$$

So, if $\lambda_{1}$ is the least non-negative integer satisfying $\left(\lambda_{1}+\theta_{1}\right) \theta>\phi$,

$$
f_{1} f_{2} \cdots f_{a_{1}}=\underbrace{00 \cdots \cdots 0}_{a_{1}-\lambda_{1}-1} 1 \underbrace{0 \cdots 0}_{\lambda_{1}} \text {. }
$$


But $\alpha_{m+1}-\alpha_{m}=a_{1}+h_{m}$. Thus if we put $h_{m}=\left[(m+1) \theta_{1}\right]-\left[m \theta_{1}\right]$, then for $m=1,2, \ldots$ and $h_{0}=0$,

$$
f_{1} f_{2} f_{3} \cdots \cdots=I_{1} I_{2} \cdots \cdots
$$

where for $m=1,2, \ldots$

$$
I_{m}=f_{\alpha_{m-1}+1} f_{\alpha_{m-1}+2} \cdots f_{\alpha_{m}}=\underbrace{00 \cdots \cdots \cdots \cdots}_{a_{1}-1-\lambda_{m}+h_{m-1}} 1 \underbrace{0 \cdots 0}_{\lambda_{m}} .
$$

Moreover,

$$
\lambda_{1}=\left[\phi / \theta-\theta_{1}\right]+1=\left[a_{1}-b_{1}+\phi_{1}\right]+1=a_{1}-b_{1}+1 .
$$

And if we put $g_{m}=h_{m}-\lambda_{m+1}+\lambda_{m}$, from $\lambda_{m}=\left[\phi / \theta-\left\{m \theta_{1}\right\}\right]+1$ we have

$$
\begin{aligned}
g_{m} & =-\left[\left(a_{1}+\theta_{1}\right) \phi-(m+1) \theta_{1}\right]+\left[\left(a_{1}+\theta_{1}\right) \phi-m \theta_{1}\right] \\
& =-\left[\theta_{1}+\phi_{1}-(m+1) \theta_{1}\right]+\left[\theta_{1}+\phi_{1}-m \theta_{1}\right] \\
& =\left[(m+1) \theta_{1}+\left(1-\theta_{1}-\phi_{1}\right)\right]-\left[m \theta_{1}+\left(1-\theta_{1}-\phi_{1}\right)\right],
\end{aligned}
$$

under the assumption $m_{1} \theta+\phi \neq m_{2}$ for any $m_{1}, m_{2} \in \mathbb{Z}$. From now on this is always assumed. Thus, we have the following theorem:

Theorem 1. Let $f_{n}=f(n ; \theta, \phi)=[(n+1) \theta+\phi]-[n \theta+\phi]-[\theta]$ and suppose the continued fraction expansion of $\theta$ is given by $\theta=\left[0, a_{1}, a_{2}, \ldots\right]=\left[0, a_{1}+\theta_{1}\right]$. For $m=1,2, \ldots$ let $g_{m}=\left[(m+1) \theta_{1}+\left(1-\theta_{1}-\phi_{1}\right)\right]-\left[m \theta_{1}+\left(1-\theta_{1}-\phi_{1}\right)\right]$.

If $0<\{\theta\}+\{\phi\}<1$, then the characteristic word is

$$
f_{1} f_{2} f_{3} \cdots=J_{0} J_{1} J_{2} J_{3} \cdots,
$$

where

$$
J_{0}=\underbrace{0 \cdots 0}_{b_{1}-2} 1, \quad J_{m}=\underbrace{00 \cdots \cdots 0}_{a_{1}-1+g_{m}} 1 \quad(m \geqslant 1) .
$$

Remark. Our discussion is quite general. When $\theta+\phi>1, n=1,2, \ldots$ the characteristic word of $f=f(n ; \theta, \phi)$ coincides with the characteristic word of $f^{\prime}=$ $f(n ; 1-\theta, 1-\phi)$ with $(1-\theta)+(1-\phi)<1$, if 0 and 1 are interchanged. When $\theta+\phi=1$, we can reduce to the homogeneous case, that is,

$$
f_{1} f_{2} f_{3} \cdots=h_{0} h_{1} h_{2} \cdots
$$

When $\theta+\phi<1, b_{1}-\phi_{1}=\phi_{0} / \theta_{0}=(1-\phi) / \theta>1$, so $b_{1} \geqslant 2$. 
We wish to rewrite this in a different way. Therefore we consider the word $g_{1} g_{2} g_{3} \cdots$ and according as $g_{m}$ is 0 or 1 we replace each $g_{m}$ by the rule

$$
g_{m}=\left\{\begin{array}{l}
0 \longrightarrow \underbrace{0 \cdots 0}_{a_{1}-1} 1=w_{1}, \text { say } \\
1 \longrightarrow \underbrace{0 \cdots 0}_{a_{1}} 1=w_{0} w_{1}
\end{array}\right.
$$

Here we have set $w_{0}=0$.

Writing $\psi(0,1)=f_{1} f_{2} f_{3} \cdots$ and $\psi_{1}(0,1)=g_{1} g_{2} g_{3} \cdots$, we have that

$$
\psi(0,1)=J_{0} \psi_{1}\left(w_{1}, w_{0} w_{1}\right)
$$

If $\theta_{1}+\phi_{1}<1$, we can continue with a similar step. This time we consider $\theta$ as $\theta_{1}, \phi$ as $1-\theta_{1}-\phi_{1}$. Then, $\theta_{1}+\left(1-\theta_{1}-\phi_{1}\right)=1-\phi_{1}<1$. If we put $\theta^{(1)}=\theta_{1}$ and $\phi^{(1)}=1-\theta_{1}-\phi_{1}$, this $\lambda_{m}\left(=\lambda_{m}^{(2)}\right.$, say $)$ is the least non-negative integer satisfying $\left(\lambda_{m}^{(2)}+\left\{m \theta_{2}\right\}\right) \theta^{(1)}>\phi^{(1)}$. Hence,

$$
\lambda_{m}^{(2)}=\left[\frac{\phi^{(1)}}{\theta^{(1)}}-\left\{m \theta_{2}\right\}\right]+1=a_{2}-b_{2}+\left[\theta_{2}+\phi_{2}-\left\{m \theta_{2}\right\}\right] .
$$

Writing $h_{m}^{(2)}=\left[(m+1) \theta_{2}\right]-\left[m \theta_{2}\right]$ and $g_{m}^{(2)}=h_{m}^{(2)}-\lambda_{m+1}^{(2)}+\lambda_{m}^{(2)}$, we obtain

$$
g_{m}^{(2)}=\left[(m+1) \theta_{2}+\left(1-\theta_{2}-\phi_{2}\right)\right]-\left[m \theta_{2}+\left(1-\theta_{2}-\phi_{2}\right)\right]
$$

and

$$
a_{2}-\lambda_{1}^{(2)}-1=b_{2}-1
$$

Thus,

$$
g_{1} g_{2} g_{3} \cdots=J_{0}^{(2)} J_{1}^{(2)} J_{2}^{(2)} J_{3}^{(2)} \cdots
$$

where

$$
J_{0}^{(2)}=\underbrace{0 \cdots 0}_{b_{2}-1} 1, \quad J_{m}^{(2)}=\underbrace{00 \cdots \cdots 0}_{a_{2}-1+g_{m}^{(2)}} 1 \quad(m \geqslant 1) .
$$

Therefore,

$$
f_{1} f_{2} f_{3} \cdots=J_{0} \psi_{1}\left(w_{1}, w_{0} w_{1}\right)=J_{0} K_{0}^{(2)} K_{1}^{(2)} K_{2}^{(2)} K_{3}^{(2)} \cdots
$$

where

$$
K_{0}^{(2)}=\underbrace{w_{1} \cdots w_{1}}_{b_{2}-1} w_{0} w_{1}, \quad K_{m}^{(2)}=\underbrace{w_{1} w_{1} \cdots \cdots w_{1}}_{a_{2}-1+g_{m}^{(2)}} w_{0} w_{1} \quad(m \geqslant 1)
$$


If $\theta_{2}+\phi_{2}<1$ again, we consider the word $g_{1}^{(2)} g_{2}^{(2)} g_{3}^{(2)} \ldots$ and the rule

$$
g_{m}^{(2)}=\left\{\begin{array}{l}
0 \longrightarrow \underbrace{0 \cdots 0}_{a_{2}-1} 1, \\
1 \longrightarrow \underbrace{0 \cdots 0}_{a_{2}} 1 .
\end{array}\right.
$$

Writing $\psi_{2}(0,1)=g_{1}^{(2)} g_{2}^{(2)} g_{3}^{(2)} \ldots$, we have

$$
\psi(0,1)=J_{0} K_{0}^{(2)} \psi_{2}\left(w_{2}, w_{1} w_{2}\right)
$$

where $w_{2}=\underbrace{w_{1} \cdots w_{1}}_{a_{2}-1} w_{0} w_{1}$. Ultimately we obtain that for $k=1,2, \ldots$,

$$
f_{1} f_{2} f_{3} \cdots=J_{0} K_{0}^{(2)} K_{0}^{(3)} \cdots K_{0}^{(k)} \psi_{k}\left(w_{k}, w_{k-1} w_{k}\right)
$$

where $w_{k}=\underbrace{w_{k-1} \cdots w_{k-1}}_{a_{k}-1} w_{k-2} w_{k-1}$ and $K_{0}^{(k)}=\underbrace{w_{k-1} \cdots w_{k-1}}_{b_{k}-1} w_{k-2} w_{k-1}$. We therefore have the following theorem:

Theorem 2. Let $f_{n}=f(n ; \theta, \phi)=[(n+1) \theta+\phi]-[n \theta+\phi]-[\theta]$ and suppose the continued fraction expansion of $\theta$ is given by $\theta=\left[0, a_{1}, a_{2}, \ldots\right]$. Let $w_{0}=0$, $w_{1}=\underbrace{0 \cdots 0}_{a_{1}-1} 1$, and $w_{k}=\underbrace{w_{k-1} \cdots w_{k-1}}_{a_{k}-1} w_{k-2} w_{k-1}$ for $k \geqslant 2$.

If $0<\{\theta\}+\{\phi\}<1$ and $\theta_{k}+\phi_{k}<1$ for every $k \geqslant 1$, then the characteristic word is

$$
f_{1} f_{2} f_{3} \cdots=\lim _{k \rightarrow \infty} J_{0} K_{0}^{(2)} K_{0}^{(3)} \cdots K_{0}^{(k)}
$$

where $J_{0}=\underbrace{0 \cdots 0}_{b_{1}-2} 1$ and $K_{0}^{(k)}=\underbrace{w_{k-1} \cdots w_{k-1}}_{b_{k}-1} w_{k-2} w_{k-1}$.

REMARK. This result matches the Main theorem of [5]. $\theta_{k}+\phi_{k}<1$ leads to $a_{k} \geqslant b_{k}$ because $1>\left(1-\phi_{k-1}\right) / \theta_{k-1}-\left(a_{k}-b_{k}\right)$ and $0 \leqslant b_{k} \leqslant a_{k}+1$. And if we write $W_{0}=w_{0}, W_{1}=w_{1}, W_{2}=W_{1}^{b_{2}-1} W_{0} W_{1}^{a_{3}-b_{2}+1}$ and $W_{k}=W_{k-1}^{b_{k}} W_{k-2} W_{k-1}^{a_{k}-b_{k}}$ for $k \geqslant 3$, then it is easily seen that for $i=1,2, \ldots$

$$
w_{2}^{i}=W_{1}^{a_{2}-b_{2}} W_{2}^{i} W_{1}^{b_{2}-a_{2}}
$$

and

$$
w_{k}^{i}=W_{1}^{a_{2}-b_{2}} W_{2}^{a_{3}-b_{3}-1} \cdots W_{k-1}^{a_{k}-b_{k}-1} W_{k}^{i} W_{k-1}^{b_{k}-a_{k}+1} \cdots W_{2}^{b_{3}-a_{3}+1} W_{1}^{b_{2}-a_{2}} \quad(k \geqslant 3)
$$


Therefore, we get $K_{0}^{(2)}=w_{1}^{b_{2}-1} w_{0} w_{1}=W_{2} W_{1}^{b_{2}-a_{2}}$ and

$$
\begin{aligned}
K_{0}^{(2)} K_{0}^{(3)} & =W_{2} W_{1}^{b_{2}-a_{2}} w_{2}^{b_{3}-1} w_{1} w_{2} \\
& =W_{2} W_{1}^{b_{2}-a_{2}} W_{1}^{a_{2}-b_{2}} W_{2}^{b_{3}-1} W_{1}^{b_{2}-a_{2}} W_{1} W_{1}^{a_{2}-b_{2}} W_{2} W_{1}^{b_{2}-a_{2}} \\
& =W_{2}^{b_{3}} W_{1} W_{2} W_{1}^{b_{2}-a_{2}}
\end{aligned}
$$

Generally for $k \geqslant 4$, from

$$
\begin{aligned}
K_{0}^{(k)}= & w_{k-1}^{b_{k}-1} w_{k-2} w_{k-1} \\
= & W_{1}^{a_{2}-b_{2}} W_{2}^{a_{3}-b_{3}-1} \cdots W_{k-2}^{a_{k-1}-b_{k-1}-1} W_{k-1}^{b_{k}-1} W_{k-2} W_{k-1} \\
& W_{k-2}^{b_{k-1}-a_{k-1}+1} \cdots W_{2}^{b_{3}-a_{3}+1} W_{1}^{b_{2}-a_{2}},
\end{aligned}
$$

we obtain

$$
K_{0}^{(2)} \cdots K_{0}^{(k)}=W_{k-1}^{b_{k}} W_{k-2} W_{k-1} W_{k-2}^{b_{k-1}-a_{k-1}+1} \cdots W_{2}^{b_{3}-a_{3}+1} W_{1}^{b_{2}-a_{2}}
$$

This $W_{k}$ is the same as the $w_{k}$ in that Main Theorem.

\section{The general cases}

We can see that $g_{m}^{(i)}$ always has the same form if $\theta_{n}+\phi_{n}<1$ for all $n$. In this section we shall show that the form of $g_{m}^{(i)}$ is always the same, even if $\theta_{k}+\phi_{k}>1$ for some $k$. We introduce $\theta_{k, l}$ and $\phi_{k, l}$ for convenience, satisfying

$$
\frac{1}{\theta_{k}}+\frac{1}{\theta_{k, l}}=l \quad \text { and } \quad \frac{\phi_{k}}{\theta_{k}}+\frac{\phi_{k, l}}{\theta_{k, l}}=l-1
$$

for an non-negative integer $l$. That is,

$$
\theta_{k, l}=-\frac{\theta_{k}}{1-l \theta_{k}} \quad \text { and } \quad \phi_{k, l}=1+\frac{\theta_{k}+\phi_{k}-1}{1-l \theta_{k}} .
$$

The case $l=1$ is known to be the necessary and sufficient condition in order that $\left\{\left[n \theta_{k}+\phi_{k}\right]\right\}_{n=1}^{\infty}$ and $\left\{\left[n \theta_{k, 1}+\phi_{k, 1}\right]\right\}_{n=1}^{\infty}$ partition the positive integers (for example, $[2])$.

Our notation allows us to write that for some integers $k$ and $l$

$$
g_{m}^{(i)}=-\left[(m+1) \theta_{k, l}+\phi_{k, l}\right]+\left[m \theta_{k, l}+\phi_{k, l}\right]
$$

3.1 CASE 1. Suppose $l=0$ and $\theta_{k}+\phi_{k}<1$. Then,

$$
g_{m}^{(i)}=\left[(m+1) \theta_{k}+\left(1-\theta_{k}-\phi_{k}\right)\right]-\left[m \theta_{k}+\left(1-\theta_{k}-\phi_{k}\right)\right]
$$


for some positive integers $i$ and $k$. By setting $\theta^{(i)}=\theta_{k}=-\theta_{k, 0}, \phi^{(i)}=1-\theta_{k}-\phi_{k}=$ $1-\phi_{k, 0}$ and $\alpha_{m}^{(i+1)}=m a_{k+1}+\left[m \theta_{k+1}\right]$, we have

$$
\begin{aligned}
\lambda_{m}^{(i+1)} & =\left[\phi^{(i)} / \theta^{(i)}-\left\{m \theta_{k+1}\right\}\right]+1 \\
& =a_{k+1}-b_{k+1}+\left[\theta_{k+1}+\phi_{k+1}-m \theta_{k+1}\right]+\left[m \theta_{k+1}\right] \\
g_{m}^{(i+1)} & =\left[(m+1) \theta_{k+1}+\left(1-\theta_{k+1}-\phi_{k+1}\right)\right]-\left[m \theta_{k+1}+\left(1-\theta_{k+1}-\phi_{k+1}\right)\right]
\end{aligned}
$$

and $a_{k+1}-\lambda_{1}^{(i+1)}-1=b_{k+1}-1$. Therefore,

$$
g_{1}^{(i)} g_{2}^{(i)} \cdots=J_{0}^{(i+1)} J_{1}^{(i+1)} J_{2}^{(i+1)} \ldots
$$

where

$$
J_{0}^{(i+1)}=\underbrace{0 \cdots 0}_{b_{k+1}-1} 1 \text { and } J_{m}^{(i+1)}=\underbrace{00 \cdots \cdots \cdots 0}_{a_{k+1}-1+g_{m}^{(i+1)}} 1 \quad(m \geqslant 1) .
$$

3.2 Case 2(1). Let

$$
g_{m}^{(i)}=-\left[(m+1) \theta_{k, l-1}+\phi_{k, l-1}\right]+\left[m \theta_{k, l-1}+\phi_{k, l-1}\right]
$$

for some positive integers $i, k$ and $l$. Suppose $\theta_{k}+\phi_{k}>1$ and $\theta_{k}<1 / l$. Put

$$
\theta^{(i)}=\theta_{k, l-1}+1=\left[0,1, a_{k+1}-l, a_{k+2}, a_{k+3}, \ldots\right] \text { and } \phi^{(i)}=\phi_{k, l-1}-1
$$

so that

$$
g_{m}^{(i)}=1-\left[(m+1) \theta^{(i)}+\phi^{(i)}\right]+\left[m \theta^{(i)}+\phi^{(i)}\right]
$$

with

$$
0<\theta^{(i)}, \phi^{(i)}, \theta^{(i)}+\phi^{(i)}<1 .
$$

If $a_{k+1} \geqslant l+1$, from $\theta^{(i)}=\left[0,1, a_{k+1}-l+\theta_{k+1}\right]$ we set $\alpha_{m}^{(i+1)}=m+\left[m\left(-\theta_{k, l}\right)\right]$.

Hence, we have

$$
\begin{aligned}
\lambda_{m}^{(i+1)} & =\left[\phi_{k, l}-1-m\left(-\theta_{k, l}\right)\right]+\left[m\left(-\theta_{k, l}\right)\right]+1 \\
g_{m}^{(i+1)} & =-\left[\phi_{k, l}-1-(m+1)\left(-\theta_{k, l}\right)\right]+\left[\phi_{k, l}-1-m\left(-\theta_{k, l}\right)\right] \\
& =-\left[(m+1) \theta_{k, l}+\phi_{k, l}\right]+\left[m \theta_{k, l}+\phi_{k, l}\right]
\end{aligned}
$$

and

$$
1-\lambda_{1}^{(i+1)}-1=-\left[\theta_{k, l}+\phi_{k, l}-1\right]-1=\left[1-\theta_{k, l}-\phi_{k, l}\right]=0 .
$$

Noticing that $\theta_{k}<1 /(l+1)$ and $\theta_{k}+\phi_{k}>1$, we remark that

$$
0<\theta_{k, l}+1, \phi_{k, l}-1, \theta_{k, l}+\phi_{k, l}<1
$$


Therefore,

$$
g_{1}^{(i)} g_{2}^{(i)} \cdots=J_{0}^{(i+1)} J_{1}^{(i+1)} J_{2}^{(i+1)} \ldots
$$

where

$$
J_{0}^{(i+1)}=0 \text { and } J_{m}^{(i+1)}=\underbrace{1 \cdots 1}_{g_{m}^{(i+1)}} 0 \quad(m \geqslant 1) .
$$

3.3. CASE 2(2). Let $g_{m}^{(i)}, \theta^{(i)}$ and $\phi^{(i)}$ be the same as those in the case 2(1). Again suppose $\theta_{k}+\phi_{k}>1$ and $\theta_{k}<1 / l$.

If $a_{k+1}=l$, from $\theta^{(i)}=\left[0, a_{k+2}+1+\theta_{k+2}\right]$ we set $\alpha_{m}^{(i+1)}=\left(a_{k+2}+1\right) m+\left[m \theta_{k+2}\right]$. Then we have

$$
\begin{aligned}
\lambda_{m}^{(i+1)}= & {\left[\phi_{k, l}-1-m \theta_{k+2}\right]+\left[m \theta_{k+2}\right]+1 } \\
g_{m}^{(i+1)}= & -\left[\phi_{k, l}-1-(m+1) \theta_{k+2}\right]+\left[\phi_{k, l}-1-m \theta_{k+2}\right] \\
= & {\left[(m+1) \theta_{k+2}+\left(1-\left(b_{k+1}+1-l\right) \theta_{k+2}-\phi_{k+2}\right)\right] } \\
& \quad-\left[m \theta_{k+2}+\left(1-\left(b_{k+1}+1-l\right) \theta_{k+2}-\phi_{k+2}\right)\right]
\end{aligned}
$$

and

$$
a_{k+2}+1-\lambda_{1}^{(i+1)}-1=a_{k+2}-\left[\phi_{k, l}-1-\theta_{k+2}\right]-1=\left[1-\theta_{k, l}-\phi_{k, l}\right]
$$

Note that

$$
0<\theta_{k+2}=-\theta_{k, l}-a_{k+2}<1
$$

and

$$
-\phi_{k, l}=-\left(b_{k+1}+1-l\right) \theta_{k+2}-\phi_{k+2}-a_{k+2}\left(b_{k+1}+1-l\right)+b_{k+2},
$$

where $b_{k+1}=l$ or $l+1$ because $\theta_{k}+\phi_{k}>1$. Therefore,

$$
g_{1}^{(i)} g_{2}^{(i)} \cdots=J_{0}^{(i+1)} J_{1}^{(i+1)} J_{2}^{(i+1)} \cdots,
$$

where

$$
J_{0}^{(i+1)}=\underbrace{11 \cdots \cdots \cdots 1}_{\left[1-\theta_{k, l}-\phi_{k, l}\right]} 0 \text { and } J_{m}^{(i+1)}=\underbrace{11 \cdots \cdots 1}_{a_{k+2}+g_{m}^{(i+1)}} 0 \quad(m \geqslant 1) .
$$

3.4. CASE 1'. Let

$$
g_{m}^{(i)}=\left[(m+1) \theta_{k}+\left(1-\left(b_{k-1}+1-l\right) \theta_{k}-\phi_{k}\right)\right]-\left[m \theta_{k}+\left(1-\left(b_{k-1}+1-l\right) \theta_{k}-\phi_{k}\right)\right]
$$

for some integers $i, k$ and $l$ with $k \geqslant 3$ and $l \geqslant 1$. From the fact in the case 2(2), $b_{k-1}=l$ or $l+1$. If $b_{k-1}=l$, we are back to case 1 . If $b_{k-1}=l+1$,

$$
g_{m}^{(i)}=\left[m \theta_{k}+\left(1-\theta_{k}-\phi_{k}\right)\right]-\left[(m-1) \theta_{k}+\left(1-\theta_{k}-\phi_{k}\right)\right]
$$


with

$$
g_{1}^{(i)}= \begin{cases}0, & \text { if } \theta_{k}+\phi_{k}<1 \\ 1, & \text { if } \theta_{k}+\phi_{k}>1\end{cases}
$$

Combining these remarks, we obtain

$$
g_{1}^{(i)} g_{2}^{(i)} \cdots=J_{-1}^{(i+1)} J_{0}^{(i+1)} J_{1}^{(i+1)} J_{2}^{(i+1)} \ldots
$$

where $J_{-1}^{(i+1)}=\underbrace{00 \cdots \cdots 0}_{b_{k-1}-l}$ if $\theta_{k}+\phi_{k}<1, \underbrace{11 \cdots \cdots 1}_{b_{k-1}-1}$ if $\theta_{k}+\phi_{k}>1$. The others are the same as those in the previous cases.

\section{TOGETHER}

Suppose that the characteristic word of the sequence $f_{n}=f(n ; \theta, \phi)$ is given by

$$
f_{1} f_{2} f_{3} \cdots=J_{0} K_{0}^{(2)} K_{0}^{(3)} \cdots K_{0}^{(i-1)} K_{1}^{(i-1)} K_{2}^{(i-1)} \cdots \text {, }
$$

where $K_{m}^{(i-1)}=u^{c+g_{m}^{(i-1)}} v$ for $m \geqslant 1$ and

$$
g_{m}^{(i-1)}=\left[(m+1) \theta_{k}+1-\left(b_{k-1}+1-l\right) \theta_{k}-\phi_{k}\right]-\left[m \theta_{k}+1-\left(b_{k-1}+1-l\right) \theta_{k}-\phi_{k}\right]
$$

for some positive integer $k$.

If $\theta_{k}+\phi_{k}<1$, then from the argument in the previous section

$$
g_{1}^{(i-1)} g_{2}^{(i-1)} \cdots=J_{-1}^{(i)} J_{0}^{(i)} J_{1}^{(i)} J_{2}^{(i)} \cdots
$$

where

$$
J_{-1}^{(i)}=\underbrace{00 \cdots \cdots 0}_{b_{k-1}-a_{k-1}}, \quad J_{0}^{(i)}=\underbrace{0 \cdots 0}_{b_{k+1}-1} 1, \quad J_{m}^{(i)}=\underbrace{00 \cdots \cdots 0}_{a_{k+1}-1+g_{m}^{(i)}} 1 \quad(m \geqslant 1),
$$

and

$$
g_{m}^{(i)}=\left[(m+1) \theta_{k+1}+\left(1-\theta_{k+1}-\phi_{k+1}\right)\right]-\left[m \theta_{k+1}+\left(1-\theta_{k+1}-\phi_{k+1}\right)\right]
$$

Therefore, we have

$$
f_{1} f_{2} f_{3} \cdots=J_{0} K_{0}^{(2)} K_{0}^{(3)} \cdots K_{0}^{(i-1)} K_{-1}^{(i)} K_{0}^{(i)} K_{1}^{(i)} K_{2}^{(i)} \cdots
$$

where

$$
K_{-1}^{(i)}=\left(u^{c} v\right)^{b_{k-1}-a_{k-1}}, \quad K_{0}^{(i)}=\left(u^{c} v\right)^{b_{k+1}-1} u^{c+1} v
$$


and for $m \geqslant 1$

$$
K_{m}^{(i)}=\left(u^{c} v\right)^{a_{k+1}-1+g_{m}^{(i)}} u^{c+1} v .
$$

In the case when $a_{k-1}=b_{k-1}, J_{-1}^{(i)}$ (so, $K_{-1}^{(i)}$ ) is omitted.

We can go to the next step by applying the substitutions

$$
u \longrightarrow u^{c} v, \quad v \longrightarrow u^{c+1} v \text { and } c \longrightarrow a_{k+1}-1 \text {. }
$$

Next, let $\theta_{k}+\phi_{k}>1$. We put $a_{k+1}=a(=l)$ and $d_{k}=\left[1-\theta_{k, l}-\phi_{k, l}\right]$. We use the argument of the previous section repeatedly.

If $a_{k+1} \geqslant 2$,

$$
g_{1}^{(i-1)} g_{2}^{(i-1)} \cdots=J_{-1}^{(i)} J_{0}^{(i)} J_{1}^{(i)} J_{2}^{(i)} \cdots
$$

where

$$
J_{-1}^{(i)}=\underbrace{11 \cdots \cdots 1}_{b_{k-1}-a_{k-1}}, \quad J_{0}^{(i)}=0, \quad J_{m}^{(i)}=\underbrace{1 \cdots 1}_{\substack{(i) \\ s_{m}}} 0 \quad(m \geqslant 1),
$$

and

$$
g_{m}^{(i)}=-\left[(m+1) \theta_{k, 1}+\phi_{k, 1}\right]+\left[m \theta_{k, 1}+\phi_{k, 1}\right]
$$

Thus, we have

$$
f_{1} f_{2} f_{3} \cdots=J_{0} K_{0}^{(2)} K_{0}^{(3)} \cdots K_{0}^{(i-1)} K_{-1}^{(i)} K_{0}^{(i)} K_{1}^{(i)} K_{2}^{(i)} \cdots,
$$

where

$$
K_{-1}^{(i)}=\left(u^{c+1} v\right)^{b_{k-1}-a_{k-1}}, \quad K_{0}^{(i)}=u^{c} v \quad \text { and } \quad K_{m}^{(i)}=\left(u^{c+1} v\right)^{g_{m}^{(i)}} u^{c} v \quad(m \geqslant 1)
$$

In the case when $a_{k-1}=b_{k-1}, J_{-1}^{(i)}\left(\right.$ so, $\left.K_{-1}^{(i)}\right)$ is omitted.

Similarly, if $a_{k+1} \geqslant a$, then

$$
g_{1}^{(i+a-3)} g_{2}^{(i+a-3)} \cdots=J_{0}^{(i+a-2)} J_{1}^{(i+a-2)} J_{2}^{(i+a-2)} \ldots,
$$

where

$$
J_{0}^{(i+a-2)}=0, \quad J_{m}^{(i+a-2)}=\underbrace{1 \cdots 1}_{g_{m}^{(i+a-2)}} 0 \quad(m \geqslant 1)
$$

and

$$
g_{m}^{(i+a-2)}=-\left[(m+1) \theta_{k, a-1}+\phi_{k, a-1}\right]+\left[m \theta_{k, a-1}+\phi_{k, a-1}\right] .
$$

Thus, we have

$$
f_{1} f_{2} f_{3} \cdots=J_{0} K_{0}^{(2)} K_{0}^{(3)} \cdots K_{0}^{(i-1)} K_{-1}^{(i)} K_{0}^{(i)} \cdots K_{0}^{(i+a-2)} K_{1}^{(i+a-2)} K_{2}^{(i+a-2)} \cdots,
$$


where

$$
K_{0}^{(i+a-2)}=u^{c} v \quad \text { and } \quad K_{m}^{(i+a-2)}=\left(u\left(u^{c} v\right)^{a-1}\right)^{g_{m}^{(i+a-2)}} u^{c} v \quad(m \geqslant 1)
$$

Finally, if $a_{k+1} \ngtr a+1$ and $a_{k+1}=a$, then

$$
g_{1}^{(i+a-2)} g_{2}^{(i+a-2)} \ldots=J_{0}^{(i+a-1)} J_{1}^{(i+a-1)} J_{2}^{(i+a-1)} \ldots,
$$

where

$$
J_{0}^{(i+a-1)}=\underbrace{1 \cdots 1}_{d_{k}} 0, \quad J_{m}^{(i+a-1)}=\underbrace{11 \cdots \cdots \cdots 1}_{a_{k+2}+g_{m}^{(i+a-1)}} 0 \quad(m \geqslant 1)
$$

and

$$
\begin{array}{r}
g_{m}^{(i+a-1)}=\left[(m+1) \theta_{k+2}+1-\left(b_{k+1}-a_{k+1}+1\right) \theta_{k+2}-\phi_{k+2}\right] \\
-\left[m \theta_{k+2}+1-\left(b_{k+1}-a_{k+1}+1\right) \theta_{k+2}-\phi_{k+2}\right]
\end{array}
$$

where $b_{k+1}=a_{k+1}$ or $a_{k+1}+1$. Thus, we have

$$
f_{1} f_{2} f_{3} \cdots=J_{0} K_{0}^{(2)} K_{0}^{(3)} \cdots K_{0}^{(i-1)} K_{-1}^{(i)} K_{0}^{(i)} \cdots K_{0}^{(i+a-1)} K_{1}^{(i+a-1)} K_{2}^{(i+a-1)} \cdots \text {, }
$$

where

$$
K_{0}^{(i+a-1)}=\left(u\left(u^{c} v\right)^{a}\right)^{d_{k}} u^{c} v, \quad K_{m}^{(i+a-1)}=\left(u\left(u^{c} v\right)^{a}\right)^{a_{k+2}+g_{m}^{(i+a-1)}} u^{c} v \quad(m \geqslant 1) .
$$

Therefore, we summarise the case when $\theta_{k}+\phi_{k}>1$, including $a_{k+1}=1$, and we have

$$
f_{1} f_{2} f_{3} \cdots=J_{0} K_{0}^{(2)} K_{0}^{(3)} \cdots K_{0}^{(i-1)} K_{-1}^{(i)} K_{0}^{(i)} K_{1}^{(i)} K_{2}^{(i)} \cdots
$$

where

$$
K_{-1}^{(i)}=\left(u^{c+1} v\right)^{b_{k-1}-a_{k-1}}, \quad K_{0}^{(i)}=\left(u^{c} v\right)^{a_{k+1}-1}\left(u\left(u^{c} v\right)^{a_{k+1}}\right)^{d_{k}} u^{c} v
$$

For $m \geqslant 1$

$$
K_{m}^{(i)}=\left(u\left(u^{c} v\right)^{a_{k+1}}\right)^{a_{k+2}+g_{m}^{(i)}} u^{c} v
$$

and

$$
\begin{aligned}
g_{m}^{(i)}=\left[(m+1) \theta_{k+2}+1-\left(b_{k+1}-\right.\right. & \left.\left.a_{k+1}+1\right) \theta_{k+2}-\phi_{k+2}\right] \\
& -\left[m \theta_{k+2}+1-\left(b_{k+1}-a_{k+1}+1\right) \theta_{k+2}-\phi_{k+2}\right],
\end{aligned}
$$

where $b_{k+1}=a_{k+1}$ or $a_{k+1}+1$. 
In the case when $a_{k-1}=b_{k-1}, J_{-1}^{(i)}\left(\right.$ so, $\left.K_{-1}^{(i)}\right)$ is omitted.

We can go to the next step by applying the substitutions

$$
u \longrightarrow u\left(u^{c} v\right)^{a_{k+1}}, \quad v \longrightarrow u^{c} v \quad \text { and } \quad c \longrightarrow a_{k+2} .
$$

Furthermore, $\theta_{k}+\phi_{k}>1$ implies $a_{k+1} \leqslant b_{k+1}$. When $a_{k+1}=b_{k+1}$, we have $d_{k}=b_{k+2}$ since $1-\theta_{k, a_{k+1}}-\phi_{k, a_{k+1}}=b_{k+2}+1-\phi_{k+2}$. When $a_{k+1}+1=b_{k+1}$, we have $d_{k}=0$ since $1-\theta_{k, a_{k+1}}-\phi_{k, a_{k+1}}=1-\left(1-\phi_{k+1}\right) / \theta_{k+1}$ and $\theta_{k}<1 / a_{k+1}$.

We obtain the following general theorem which incorporates Theorem 2:

Theorem 3. Let $\theta$ be irrational and $\phi$ be real, satisfying $0<\theta, \phi, \theta+\phi<1$ and $m_{1} \theta+\phi \neq m_{2}$ for any $m_{1}, m_{2} \in \mathbb{Z}$. Then the characteristic word of the sequence $f_{n}=f(n ; \theta, \phi)$ is given by

$$
f_{1} f_{2} f_{3} \cdots=\lim _{n \rightarrow \infty} J_{0} J_{k_{1}} J_{k_{2}} J_{k_{3}} \cdots J_{k_{n}} .
$$

Here, $k_{1}, k_{2}, k_{3}, \ldots$, are determined by

$$
k_{1}=1, \quad k_{n+1}=\left\{\begin{array}{ll}
k_{n}+1, & \text { if } \theta_{k_{n}}+\phi_{k_{n}}<1 ; \\
k_{n}+2, & \text { if } \theta_{k_{n}}+\phi_{k_{n}}>1
\end{array} \quad(n \geqslant 1) ;\right.
$$

and $J_{0}=\underbrace{0 \cdots 0}_{b_{1}-2} 1$. For $k=k_{1}, k_{2}, k_{3}, \ldots$, if $\theta_{k}+\phi_{k}<1$,

$$
J_{k}=\underline{u_{k+1}^{b_{k-1}-a_{k-1}} u_{k+1}^{b_{k+1}-1} v_{k+1},}
$$

where $u_{1}=0, v_{1}=1$, and for $k=k_{n} \geqslant 1$

$$
u_{k+1}=\left\{\begin{array}{ll}
u_{k}^{a_{k}-1} v_{k}, & \text { if } k_{n-1}=k-1 ; \\
u_{k}^{a_{k}} v_{k}, & \text { if } k_{n-1}=k-2,
\end{array} \quad v_{k+1}=u_{k} u_{k+1} .\right.
$$

If $\theta_{k}+\phi_{k}>1$,

$$
J_{k}=\underline{\left(u_{k} v_{k+2}\right)^{b_{k-1}-a_{k-1}}} v_{k+2}^{a_{k+1}-1} u_{k+2}^{d_{k}} v_{k+2},
$$

where $u_{1}=0, v_{1}=1$, and for $k=k_{n} \geqslant 1$

$$
\begin{gathered}
u_{k+2}=u_{k} v_{k+2}^{a_{k+1},} \quad v_{k+2}= \begin{cases}u_{k}^{a_{k}-1} v_{k}, & \text { if } k_{n-1}=k-1 ; \\
u_{k}^{a_{k}} v_{k}, & \text { if } k_{n-1}=k-2 .\end{cases} \\
d_{k}= \begin{cases}0, & \text { if } a_{k+1}+1=b_{k+1} ; \\
b_{k+2}, & \text { if } a_{k+1}=b_{k+1} .\end{cases}
\end{gathered}
$$

The underlined parts occur as stated when $k_{n}=k(\geqslant 3)$ and $k_{n-1}=k-2$. Otherwise, they are empty. 


\section{EXAMPLE}

Let $\theta=\sqrt{3}-1$ and $\phi=\sqrt{5}-2$. Then

$$
\begin{array}{r}
a_{1}=1, a_{2}=2, a_{3}=1, a_{4}=2, a_{5}=1, a_{6}=2, a_{7}=1, a_{8}=2, a_{9}=1, \ldots \\
b_{1}=2, b_{2}=3, b_{3}=1, b_{4}=2, b_{5}=1, b_{6}=1, b_{7}=2, b_{8}=3, b_{9}=2, \ldots
\end{array}
$$

and

$$
\begin{gathered}
\theta+\phi, \theta_{3}+\phi_{3}, \theta_{5}+\phi_{5}, \theta_{10}+\phi_{10}, \ldots<1 \\
\theta_{1}+\phi_{1}, \theta_{2}+\phi_{2}, \theta_{4}+\phi_{4}, \theta_{6}+\phi_{8}, \theta_{7}+\phi_{7}, \theta_{8}+\phi_{8}, \theta_{9}+\phi_{9}, \ldots>1
\end{gathered}
$$

So, $d_{1}=0, d_{2}=2, d_{4}=1, d_{6}=0, d_{7}=0, d_{8}=0, \ldots$

Therefore,

$$
f_{1} f_{2} f_{3} \cdots=J_{0} J_{1} J_{3} J_{4} J_{6} \cdots,
$$

where $J_{0}=1, J_{1}=v_{3}^{a_{2}-1} u_{3}^{d_{1}} v_{3}=11$ from $v_{3}=u_{1}^{a_{1}-1} v_{1}=1$ and $u_{3}=u_{1} v_{3}^{a_{2}}=011$, $J_{3}=u_{4}^{b_{2}-a_{2}} u_{4}^{b_{4}-1} v_{4}=011101110110111$ from $u_{4}=u_{3}^{a_{3}} v_{3}=0111$ and $v_{4}=u_{3} u_{4}=$ 0110111 ,

$$
\begin{aligned}
& J_{4}=v_{6}^{a_{5}-1} u_{6}^{d_{4}} v_{6}=01110111011011101110110111, \\
& J_{6}=v_{8}^{a_{7}-1} u_{8}^{d_{6}} v_{8}=01110111011011101110111011011101110110111,
\end{aligned}
$$

because $v_{6}=u_{4}^{a_{4}-1} v_{4}=01110110111$ and $u_{6}=u_{4} v_{6}=011101110110111, v_{8}=$ $u_{6}^{a_{6}} v_{8}=01110111011011101110111011011101110110111$ and $u_{8}=u_{6} v_{8}^{a_{7}}$.

\section{REFERENCES}

[1] L.V. Danilov, 'Some class of transcendental numbers', Mat. Zametki 12 (1972), 149-154. Math. Notes 12 (1972), 524-527.

[2] A.S. Fraenkel, 'The bracket function and complementary sets of integers', Canad. J. Math. 21 (1969), 6-27.

[3] A.S. Fraenkel, M. Mushkin and U. Tassa, 'Determination of [n $\theta]$ by its sequence of differences', Canad. Math. Bull. 21 (1978), 441-446.

[4] Sh. Ito and S. Yasutomi, 'On continued fractions, substitutions and characteristic sequences $[n x+y]-[(n-1) x+y]$ ', Japan. J. Math. 16 (1990), 287-306.

[5] T. Komatsu, 'A certain power series associated with Beatty sequences', (Manuscript in preparation).

[6] T. Komatsu, 'The fractional part of $n \theta+\phi$ and Beatty sequences', J. Théorie des Nombres de Bordeaux (to appear).

[7] K. Nishioka, I. Shiokawa and J. Tamura, 'Arithmetical properties of certain power series', J. Number Theory 42 (1992), 61-87. 
[8] T. van Ravenstein, G. Winley and K. Tognetti, 'Characteristics and the three gap theorem', Fibonacci Quart. 28 (1990), 204-214.

[9] K.B. Stolarsky, 'Beatty sequences, continued fractions, and certain shift operators', Canad. Math. Bull. 19 (1976), 473-482.

[10] B.A. Venkov, Elementary number theory (Wolters-Noordhoff, Groningen, 1970).

\author{
Centre for Number Theory Research \\ Macquarie University \\ New South Wales 2109 \\ Australia \\ e-mail: komatsu@mpce.mq.edu.au
}

